\title{
Research on the Design of SINS/GPS Integrated Navigation System Ruimin Zhang ${ }^{1, a}$, Xusheng Wang ${ }^{2, b}$ and Shigang Fan ${ }^{3, c}$ \\ ${ }^{1,2,3}$ High-tech Institute,Fan Gong-ting South Street on the 12th, QingZhou 262500 , China.
}

Keywords: SINS; GPS; Integrated navigation system

\begin{abstract}
The functional design is carried out from global positioning, navigation instructions and other aspects based on the navigation system design. However, in the process of using SINS/GPS combination, it needs to be studied from the aspects of operation precision, system operability and so on. On the premise of analyzing the advantages and disadvantages of SINS/GPS combination, we discuss the design and application of navigation system, aiming to provide reference for future navigation system design.
\end{abstract}

\section{Introduction}

As an irreplaceable excellent navigation system in the military field, SINS's (strapdown inertial navigation) anti-interference ability, high work autonomy and high output information are all better than other similar systems. But the only drawback is that once the running time is long, the accuracy will continue to decline. At present, there are two ways to improve the SINS accuracy. The first one is to improve the accuracy of the inertial navigation system itself. The second one is to combine inertial navigation and other navigation sensors to form a combined navigation system. By contrast, the combined system is easier to implement, because the improvement of INS is based on the new process, new technology and new materials, and the cost of manpower and material resources is much higher than that of combined navigation.

\section{The Meaning of the Combination of SINS and GPS}

SINS and GPS have their own good points and they complement each other, therefore, the combination of SINS and GPS can give full play to the advantages of the two, and avoid the disadvantages. The advantages of high precision of long-term running of GPS can be used to make up the shortcomings of the large error of long-term running of SINS. In the same way, the advantages of SINS can also make up the shortcomings of GPS. The combination of the two can achieve a better navigation effect[1].

\section{Integrated Navigation Design Scheme}

The whole system uses the 'DSP+GPS-OEM board +MINU' architecture, and the details are shown in figure 1:

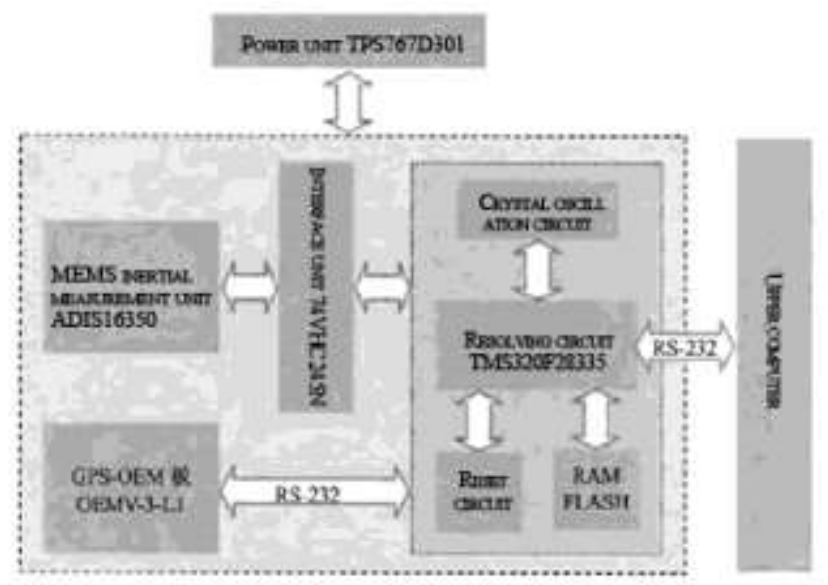

Figure 1. System Framework 
The information of the linear velocity, angular velocity, acceleration and pseudo-range rate of the carrier movement is provided by the inertial surveyor and GPS-OEM to the calculation unit respectively. The operation of attitude, speed, state and estimated mean square error is carried out by the calculation unit that receives the information, and the latitude and longitude and the angle of the attitude are obtained. In addition, the interface unit has to carry out the level conversion to complete the data communication between the calculation unit and the sensor. This is because the output voltage and the input voltage of the interface between the calculation unit and the sensor are different. Finally, the attitude is communicated with the host computer through the RS232 serial port module.

\section{Inertial Measurement Module}

The space inside the projectile is small, so the medium precision MEMS inertial sensor ADIS16350 should be selected as the measuring unit. ADIS16358 has high precision, and small size, which can meet the space $\operatorname{limitation}(22.7 * 23.2 * 22.9$ unit: $\mathrm{mm})$, and it integrates a three-axis accelerometer and a three-axis gyroscope, which can provide the original data to the projectile attitude. The internal principles are shown in figure 2:

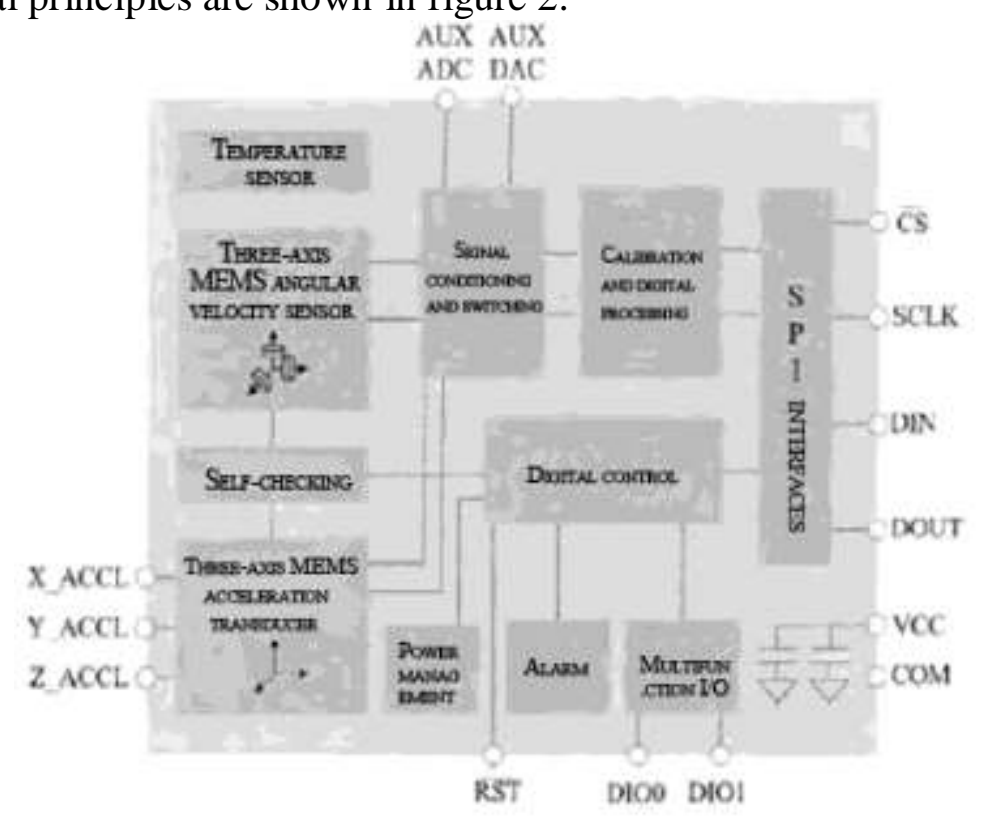

\section{GPS Part}

Figure 2. ADIS16358 schematic diagram

The OEMV-3-L1/GPS-OEM board in Novtel is selected(88*57*15 unit: mm), as shown in figure 3. The weight of OEMV-3-L1 is $48 \mathrm{~g}$, which is suitable for microminiature navigation to realize inertial navigation system integration. The parameters of OEMV-3-L1: The power supply voltage is $4.5-\mathrm{v} 18 \mathrm{~V}$, power consumption is $2.1 \mathrm{~W}$, the single point positioning accuracy is $1.5 \mathrm{~m}$, the speed is $0.03 \mathrm{~m} / \mathrm{s}$ RMS, and the data update rate is $20 \mathrm{~Hz}$, as shown in figure 3 .

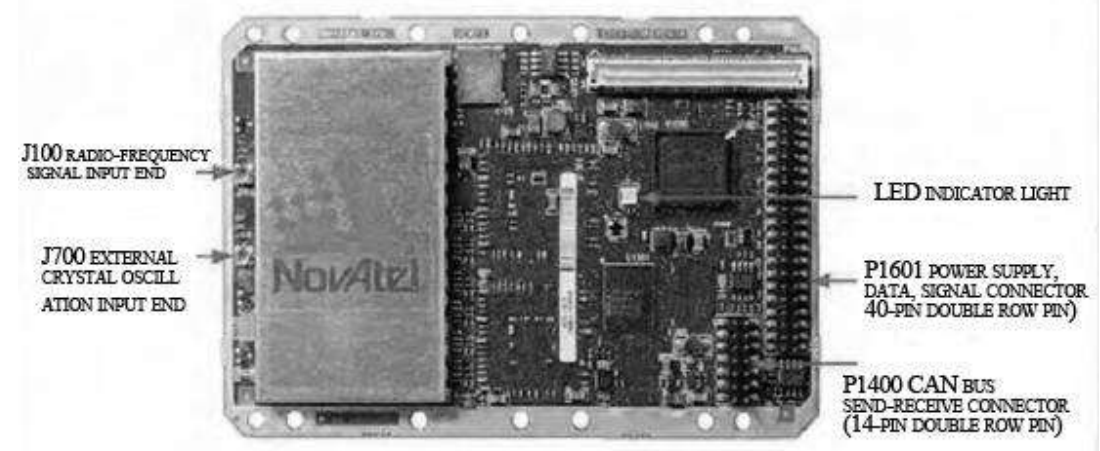

Figure 3. OEMV-3-L1 


\section{Interface Unit}

ADIS16350 provides four-line SPI interface, as shown in figure 2. The GPIO pin level logic of the TMS329F28335 is 3.3V, but the pin level logic of ADIS16360 is 5V, so only by using $74 \mathrm{VHC} 245 \mathrm{~N}$ chip to achieve level conversion between them, can DAIS16350 achieve communication function with processor TM320F28335, as shown in figure 4 .

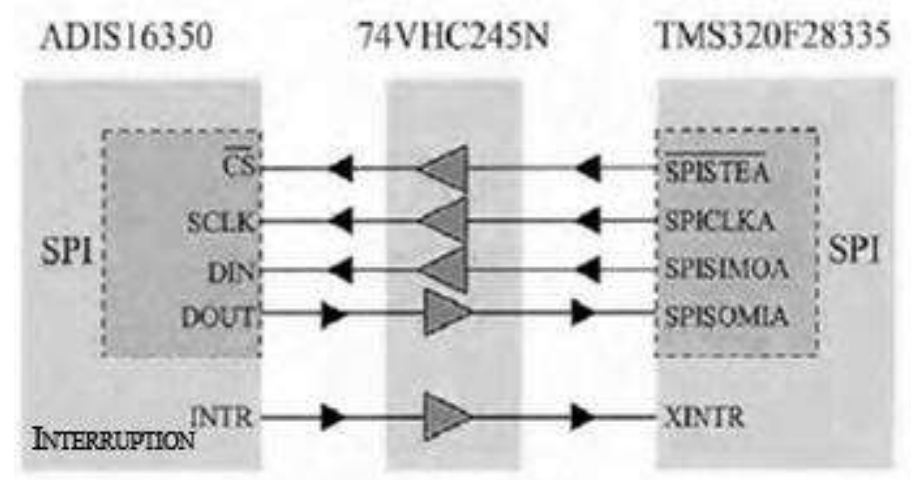

Figure 4. Interface circuit diagram

\section{Attitude Calculation Processor and Peripheral Circuit}

The prediction operation, valuation operation, and estimated mean square error operation of the attitude are all performed by the attitude calculation processor, which involves the high order matrix operation process. Most of the variables in the system, including matrix elements, are all 64-bit single-precision floating point. The accuracy and speed of its operation are very high, and the calculation frequency must reach $100 \mathrm{~Hz}$. Therefore, the DPS controller selects the TMS320F 28335 type produced by TI, and the controller data is a 32-bit floating-point database. The circuit diagram is shown in figure 5.

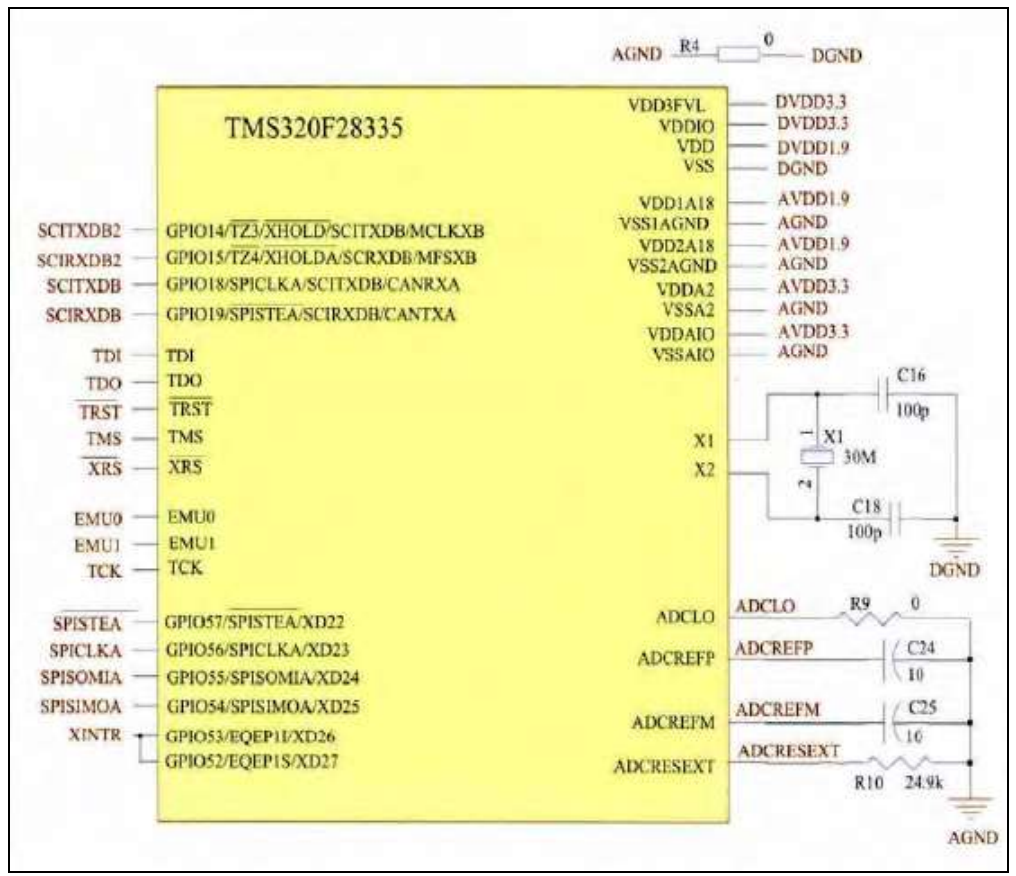

Figure 5. The partial pin diagram and peripheral circuit connection diagram of TMS320F283235 attitude solver

There is the single-precision floating-point operation unit FPU of composite IEEE-754 in TMS320F28335 chip. Both the $16 * 16$ and the $32 * 32$ bit multiplication accumulator operations can be carried out. It allows instruction and data extraction to run at the same time (improved Harvard pipeline structure), which can quickly interrupt response. The digital crystal oscillator and watchdog module are integrated in the chip, and the clock system supports the dynamic phase-locked loop to 
adjust frequency doubling. At the same time, the peripherals include serial communication interface module (SCI module) *3, 32-bit CPU timer *3, CAN bus module *2, McBSP (channel buffered port) $* 2$, a SPI interface module and 16 channels 12-bit ADC, and 87 general I/Os (GPIO) can be selected as the input and output of various peripherals. These rich peripherals can satisfy the user's simple and convenient design of the circuit in the engineering practice, and have a great contribution to the integration of the system.

For the simplicity of the design, the clock circuit uses the TMS320F28335 internal crystal oscillator, the external $30 \mathrm{MHz}$ crystal oscillator and a programmable phase-locked loop. The user can set up in a programmable phase-locked loop according to the clock of the required system.

\section{Power Module}

20F28335 is divided into kernel power supply (1.9V) and GPIO power supply (3.3V). The power unit uses TPS767D301 two circuit voltage output devices, and uses external 5V lithium battery to generate voltage of $1.9 \mathrm{~V}$ and $3.3 \mathrm{~V}$, and it can be used as the source of reset signal of TMS320F28335 processor, as shown in figure 6.

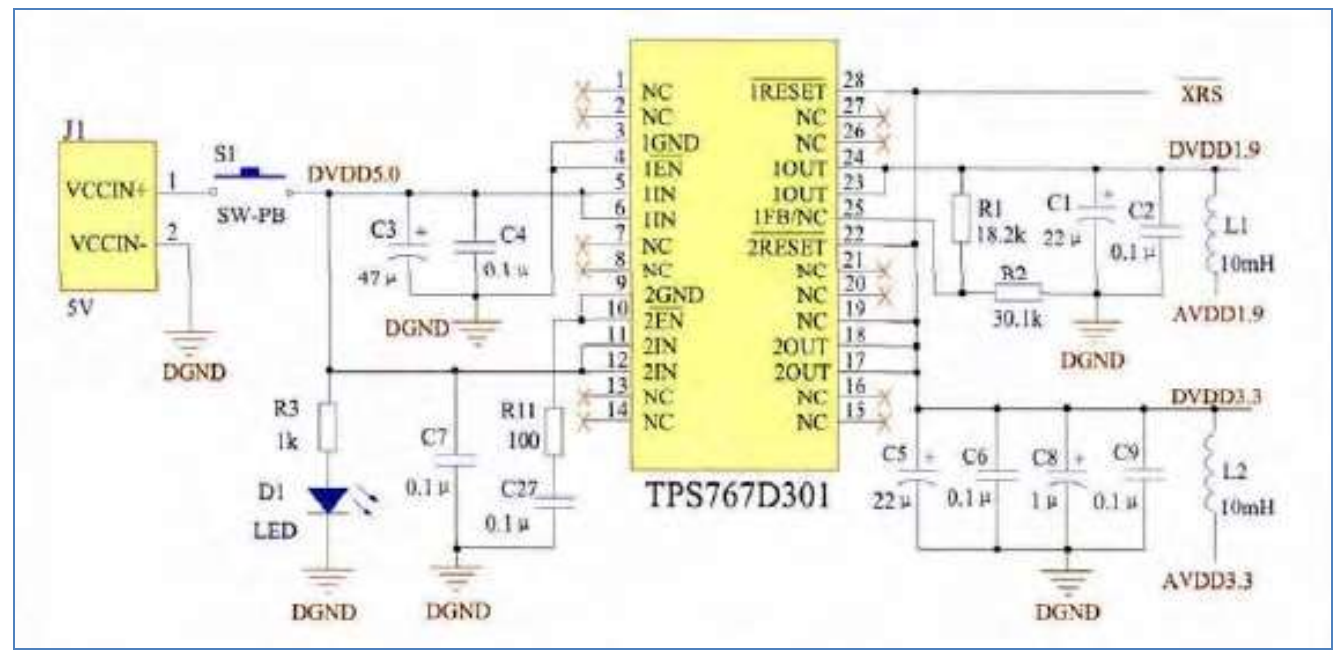

Figure 6. Power circuit connection diagram (part)

\section{Serial Port Unit}

The system requires two RS-232 serial communication interfaces. The first RS-232 is responsible for establishing communication with the host computer for real-time monitoring of attitude calculation. The second RS-232 needs to communicate with the GPS-OEM card, as shown in figure $7[2]$ :

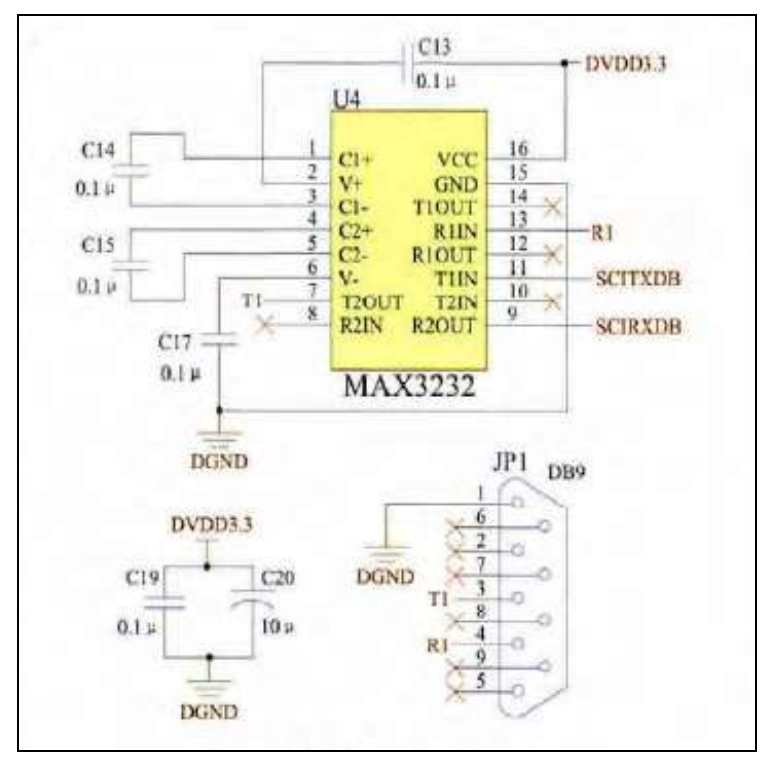

Figure 7. Serial connection diagram 


\section{Software Design}

The system adopts the modular design method to design the software part. According to the characteristics of the attitude system software, it is divided into six modules: initialization of software and hardware, initialization of combined attitude measurement system, data acquisition of inertial sensors, acquisition of GPS data, attitude calculation and filtering, and data output. The C language is used for programming. Then the compilation, debugging and testing of each module are completed, and the software implementation of the whole system is completed.

\section{Experiment}

Testing system performance in a high dynamic environment can be performed with the aid of three-axis turntable equipment. Firstly, we started the three-axis turntable, and let it simulate the maneuvering flight state of the UAV, and the measured value was accurate[3]. Then, we reasonably fixed the optimized attitude performance system on the three-axis turntable, and made the corresponding simulation for the rotational speed according to the aerodynamic simulation experiment, so as to control the rotation of the turntable. At the same time, the filtering performance of the system and the calculation accuracy of the attitude angle are analyzed by comparing the attitude angle obtained by the system calculation and the real attitude angle of the experiment of the turntable. The results show that the initial state of the attitude angle is: $\theta=7.7^{\circ} \gamma=0^{\circ} \varphi=149^{\circ}$, and the overall test time is $5.8 \times 104 \mathrm{~s}$. Through the above analysis, the attitude angle curves are obtained as shown in the following figure. The details are shown in figure $\mathrm{X}$.

\section{Conclusion}

Through the above experiment, we can clearly see that the filtering of the pitch angle, the roll angle, and the course angle is in a state of instability when the system is in the initial work, which leads to a great error in the estimated attitude value and the true value. However, with the stability of the system work, the error of the attitude angle converges gradually, which can satisfy the real attitude of the UAV. When the time is lengthening for about 2 minutes, it can be in line with the real attitude of the UAV. At the same time, the system can also effectively realize the attitude tracking of the free rotation of the yaw angle, and a better transition can be achieved at the $360^{\circ}$ critical point.

The experimental results of dynamic turntable show that the designed attitude measurement system can well restrain the attitude divergence caused by gyroscopic drift in the state of the high maneuverable flight in the flying body, and can achieve the true attitude of a high precision tracking flight body, so as to achieve the perfect navigation[4].

\section{References}

[1] Jialiang Pan, Zhi Xiong, Lina Wang, etc: A Simplified UKF Algorithm for SNS/GPS/CNS Integrated Navigation System in Launch Inertial Coordinate System[J], Acta Armamentarii, 2015, 36(3):484-491.

[2] Zhi Xiong, Jialiang Pan, Aijun Lin, etc: SINS/GPS/CNS Integrated Navigation System Federal PF Algorithm in Launch Coordinate System [J], Journal of Nanjing University of Aeronautics \& Astronautics, 2015, 47(3):319-323.

[3] Fei Wang: Research on SINS/GPS Integrated Navigation System[D], Beijing Institute of Technology, 2015.

[4] Lei Wang, Fengfeng Shi, Kaiyang Kou, etc: Modeling and Simulation of Unmanned Aerial Vehicle b (UAV) Integrated Navigation System Based on SINS/GPS[J], Engineering of Surveying and Mapping, 2016, 25(10):17-19. 